\title{
Are services meeting the holistic family planning needs of female sex workers living with HIV in Tanzania?
}

Project SOAR

Follow this and additional works at: https://knowledgecommons.popcouncil.org/departments_sbsr-hiv

Part of the Demography, Population, and Ecology Commons, Family, Life Course, and Society Commons, and the International Public Health Commons How does access to this work benefit you? Let us know!

\section{Recommended Citation}

Project SOAR. 2018. "Are services meeting the holistic family planning needs of female sex workers living with HIV in Tanzania?" Project SOAR Activity Brief. Washington, DC: Population Council. 


\section{Are Services Meeting the Holistic Family Planning Needs of Female Sex Workers Living with HIV in Tanzania?}

Women of reproductive age living with HIV need access to comprehensive, voluntary family planning (FP) services to support their decisions about whether and when to have children. Contraceptive need is particularly acute among HIV-positive female sex workers who are often socially and economically disadvantaged. Furthermore, many HIV-positive female sex workers intend to have children some day, but may not be aware of strategies to minimize the risk of HIV transmission to infants and partners (i.e., "safer conception").

In Tanzania, Project SOAR is contributing to the evidence base by conducting research that describes these women's FP needs and fertility intentions. We are also examining ongoing community-based FP services for HIVpositive women who sell sex. In addition, we are documenting the knowledge and opinions around strategies for conception among health

Research Partners: Population Council; Jhpiego's Sauti Project; CSK Research Solutions; Ministry of Health Community Development, Gender, Elderly and Children

Location: Dar es Salaam

Study Duration: 2017-2018

For more information, contact Eileen Yam, Population Council, eyam@popcouncil.org.

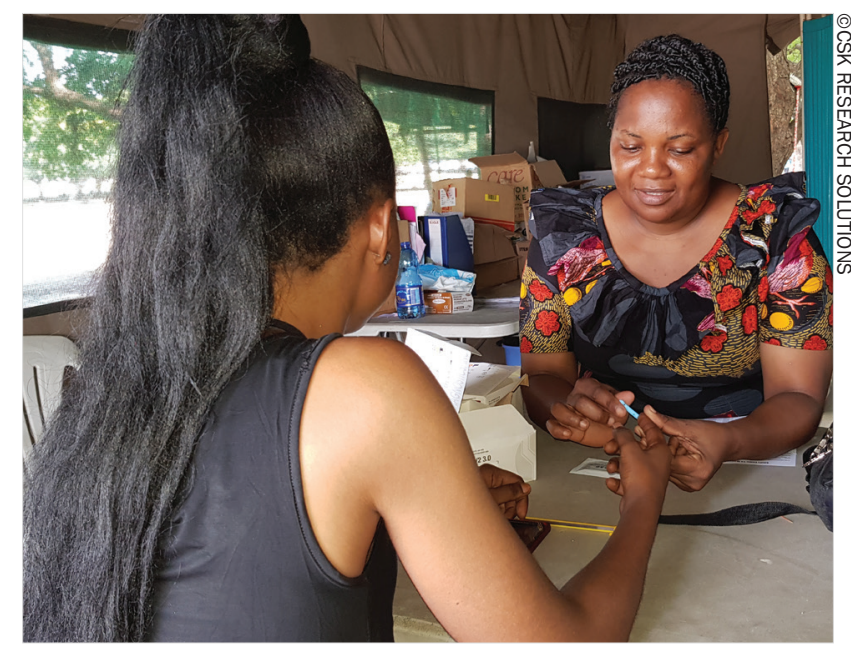

Findings from this study will help inform national and global guidance on the provision of family planning and "safer conception" counseling services.

providers and female sex workers living with HIV. Although there are various strategies for reducing vertical and sexual HIV transmission when trying to conceive, it is unclear the extent to which providers and HIV-positive women find these options acceptable or feasible.

In Tanzania, national guidelines recommend providing FP and "safer pregnancy" counseling services for women living with HIV, as well as integrated reproductive health services for female sex workers. This research will help inform national and global guidance on the provision of both types of services to HIV-positive women, thereby reducing mother-to-child HIV transmission and helping women decide whether and when to have children. 


\section{What is "safer conception?"}

Safer conception refers to strategies to help HIV-affected individuals and couples get pregnant while minimizing the risk of HIV transmission to HIV-negative partners and the baby. Low- and high-technology safer conception strategies include timed unprotected intercourse, self-insemination with sperm of the HIV-negative partner, and biomedical interventions such as PrEP-or pre-exposure prophylaxis-for the HIV-negative partner and antiretroviral therapy for the HIVpositive partner.

\section{Our Research}

We are conducting this study at selected community-based sites in Dar es Salaam where Jhpiego's Sauti Project offers services to female sex workers.

The study objectives are to:

- Describe HIV-positive female sex workers' fertility-related needs (e.g., contraceptive need, fertility intentions, dual method use and acceptability).

- Describe Sauti's ongoing community-based FP services to HIV-positive female sex workers.

- Explore awareness, accessibility, and acceptability of safer conception strategies among Sauti's clients and healthcare providers.

SOAR is conducting quantitative client exit interviews with 300 HIV-positive female sex workers, qualitative interviews with 10 Sauti providers and 15 women who are currently trying to get pregnant, and 25 structured observations of women's consultations with providers.

By exploring both providers' and women's perspectives, this study will produce valuable insights to inform future efforts to support the holistic FP needs of HIV-positive women in Tanzania and other developing countries.

\section{Sauti community-based services}

The PEPFAR-supported Sauti project provides an integrated set of FP and HIV services for key populations in discreet and safe locations throughout Tanzania. These include HIV testing services, gender-based violence services, sexually transmitted infection screening and treatment, alcohol and drug abuse screening, and FP counseling and methods (all modern methods except for intrauterine devices or tubal ligation, for which Sauti provides referrals). In communities, peer educators generate demand for Sauti services, encouraging peers to attend these key population-friendly, community-based services.

\section{Research Utilization}

This study is of interest to stakeholders in both the HIV and reproductive health arenas, and we will identify and convene a research advisory committee (RAC) that represents both constituencies. Furthermore, the Population Council enjoys a strong international presence as a technical leader in the cross-cutting thematic areas of reproductive health, HIV, and gender. Leveraging Council staff's global professional networks and communities of practice, the study team will seek out opportunities to disseminate and promote utilization of study findings among stakeholders who promote HIV and FP integration.
Project SOAR/Population Council

4301 Connecticut Avenue, NW, Suite 280

Washington, DC 20008

Tel: +12022379400

e-mail: ProjectSOAR@popcouncil.org

projsoar.org

(C)Population Council, April 2018 